\title{
Le Maroc face au défi des réfugiés économiques : quelle approche pour quelle gouvernance migratoire?
}

The Challenge of Economic Refugees in Morocco: What Approach for which

Migration Governance?

Marruecos ante el reto de los refugiados económicos: ¿Qué aproximación para

qué gobernanza migratoria?

Brahim Elmorchid et Hind Hourmat-Allah

\section{(2) OpenEdition}

\section{Journals}

Édition électronique

URL : https://journals.openedition.org/remi/11216

DOI : $10.4000 /$ remi. 11216

ISSN : $1777-5418$

Éditeur

Université de Poitiers

Édition imprimée

Date de publication : 1 octobre 2018

Pagination : 229-252

ISBN : 979-10-90426-62-7

ISSN : 0765-0752

Référence électronique

Brahim Elmorchid et Hind Hourmat-Allah, « Le Maroc face au défi des réfugiés économiques : quelle approche pour quelle gouvernance migratoire? », Revue européenne des migrations internationales [En ligne], vol. 34 - n² et 3 | 2018, mis en ligne le 01 janvier 2021, consulté le 14 avril 2022. URL : http:// journals.openedition.org/remi/11216; DOI : https://doi.org/10.4000/remi.11216 


\section{Le Maroc face au défi des réfugiés économiques : quelle approche pour quelle gouvernance migratoire? \\ Brahim Elmorchid ${ }^{1}$ et Hind Hourmat Allah ${ }^{2}$}

Les déplacements des populations suscités par la pauvreté, la dégradation des conditions de vie et l'injustice économique ne sont pas nouveaux. Tout au long de l'histoire, les hommes ont fait preuve d'une forte capacité d'adaptation et de résilience face aux conditions économiques désastreuses imputables à différents facteurs : ralentissement économique, creusement du déficit public, pression fiscale, aléas climatiques, etc. Aussi, au cours de ces dernières années, les crises économiques sous toutes leurs formes sont de plus en plus fréquentes, de plus en plus graves et leurs conséquences sociales sont de plus en plus néfastes, si bien qu'un nouveau type de migrants apparait sur la scène internationale : "les réfugiés économiques".

Les "réfugiés économiques" sont des personnes qui migrent contre leur gré en raison d'une persécution économique ou d'une privation économique sévère qui affectent profondément leur qualité de vie et met en péril leur existence. En 2017, et d'après les estimations des Nations unies, le nombre de migrants dans le monde a atteint 258 millions contre 154,2 millions enregistrés en 1990 (United Nations, 2017 : 4). Toutefois, seuls 22,5 millions, soit $9 \%$, sont éligibles au statut de réfugié tel qu'il est défini par la Convention de Genève (UNHCR, 2017 : 2). Les autres migrants sont qualifiés par le Haut Commissariat des Nations unies pour les réfugiés (UNHCR) de simples "migrants économiques", car ils sont soupçonnés de faire le choix de se déplacer à la recherche de meilleures perspectives matérielles pour eux-mêmes et leurs familles (UNHCR, 2016). Pourtant, nombre d'entre eux ont été forcés de quitter leur pays d'origine afin d'échapper à une extrême pauvreté due, entre autres, aux persécutions de nature économique (privation des terres, violation des droits économiques, défaillance du marché de travail, etc.).

Malgré la gravité et la complexité de ce phénomène, seules des réponses ponctuelles et essentiellement humanitaires furent proposées par les acteurs concernés (les gouvernements, les agences des Nations unies et les ONG). La

\footnotetext{
1 Enseignant-chercheur, département d'économie, Faculté de Droit, Université cadi Ayyad, BP 2380, Daoudiate, Marrakech, Maroc; morchid_brahim@yahoo.fr

2 Enseignante-chercheuse, département des sciences de gestion, Faculté de Droit, Université cadi Ayyad, BP 2380, Daoudiate, Marrakech, Maroc; hourmat_hind@yahoo.fr
} 
communauté internationale semble incapable d'agir afin d'élaborer une solution globale, participative et durable. On constate que les droits et la dignité des "réfugiés économiques" sont souvent bafoués dans les sociétés d'immigration. Des termes à caractère déshumanisant comme "immigrés illégaux», "immigrés clandestins" ou encore "immigrés sans-papiers" sont fréquemment employés pour qualifier cette catégorie de migrants non désirés. Or, ces dénominations péjoratives ne semblent pas innocentes. Leur utilisation reflète une certaine discrimination de ces personnes vulnérables et la montée croissante d'un sentiment anti-immigration dans les pays d'arrivée.

En dépit de ce constat inquiétant, les réflexions académiques sur la caractérisation et la protection des "réfugiés économiques" ne sont pas encore suffisamment étayées. Or de telles réflexions semblent cruciales tant pour mieux cerner ce concept que pour sensibiliser les différentes parties prenantes aux enjeux humanitaires et sécuritaires que représente l'asile économique pour les sociétés d'arrivée. C'est donc pour contribuer au débat sur cette question que nous avons jugé opportun d'étudier le cas marocain. Le choix de ce terrain de recherche est justifié par le fait que depuis le début du XXle siècle, le Maroc est devenu à la fois une terre d'accueil et de transit pour des milliers de réfugiés économiques originaires d'Afrique subsaharienne.

Notre propos est organisé comme suit. Dans la première section, nous tenterons de définir et de caractériser le terme de "réfugié économique". Dans la deuxième section, nous montrerons qu'en dépit de son importance, la question de I'asile économique au Maroc est très peu élucidée. Dans la troisième section, nous analyserons la place de la dimension économique dans la politique d'immigration et d'asile au Maroc. En conclusion, nous reviendrons sur les résultats les plus saillants et nous nous interrogerons sur les implications en termes de politiques économiques.

\section{Les réfugiés économiques : de qui parle-t-on?}

Depuis le début du XXle siècle, le terme "réfugié économique" s'est progressivement imposé dans les discours médiatiques, politiques et académiques. Pourtant, pour les juristes, ce terme n'a aucune base légale et il relève même de ce que certains experts en droit appellent "l'erreur juridique" (McAdam, 2009). Les conventions internationales et les lois nationales ne font aucune référence aux critères économiques dans la définition du terme "réfugié". Seuls les critères politiques et sécuritaires sont retenus.

\section{La dimension économique dans les principales Conventions sur les réfugiés : un essai d'interprétation}

Plusieurs textes et déclarations internationaux ont tenté de clarifier le sens véhiculé par le mot "réfugié ». Citons à titre d'exemples la Convention de Genève de 1951, le Protocole relatif au statut des réfugiés de NewYork du 31 janvier 1967, la Convention de I'Organisation de I'Unité africaine (OUA) régissant les aspects propres aux problèmes des réfugiés en Afrique et la Déclaration de Carthagène sur les réfugiés de 1984. Ainsi, d'après l'article premier de la Convention de Genève du 24 juillet 1951 relative au statut des réfugiés, un réfugié est défini comme "une personne qui fuit son pays parce qu'elle a peur d'être persécutée 
du fait de sa race, de sa religion, de sa nationalité, de son appartenance à un groupe social déterminé ou de ses opinions politiques" (HCR, 2007 : 16). Cette définition assez large ouvre la voie à deux interprétations complémentaires dans le sens de la reconnaissance des réfugiés économiques.

D'un côté, la Convention de Genève a retenu, entre autres critères, l'appartenance à un groupe social comme espace de persécution. Or aucune définition précise n'a été attribuée à ce concept, ce qui pourrait donner lieu à des interprétations diverses et à des positions divergentes dans le milieu juridique. Un groupe social est généralement reconnu par deux éléments : I'existence d'interactions entre les membres et la conscience d'une appartenance commune. Ceci ouvre donc logiquement la porte à des groupes sociaux issus des pays pauvres et composés de personnes économiquement très défavorisées, de personnes victimes de catastrophes naturelles ou encore de personnes exclues du système économique, de figurer dans le champ de définition des réfugiés au sens de la Convention en question.

D'un autre côté, le préambule de la Convention de Genève fait explicitement référence à la Déclaration universelle des Droits de l'Homme de 1948. Or celle-ci énonce dans son article 25 que "toute personne a droit à un niveau de vie suffisant pour assurer sa santé, son bien-être et ceux de sa famille, notamment pour l'alimentation, I'habillement, le logement, les soins médicaux ainsi que pour les services sociaux nécessaires" (HCR, 2007 : 29). Dans ces conditions, il semble que la construction d'un statut pour les "réfugiés économiques" n'est pas contraire à l'esprit de cette Convention.

Si nous considérons maintenant la Convention de l'Organisation de I'Unité africaine (OUA), qui régit les aspects propres aux problèmes des réfugiés en Afrique et dont le Maroc est l'un des signataires en 1969, elle étend la définition du réfugié à "toute personne qui, du fait d'une agression, d'une occupation extérieure, d'une domination étrangère ou d'événements troublant gravement l'ordre public dans une partie ou dans la totalité de son pays d'origine ou du pays dont elle a la nationalité, est obligée de quitter sa résidence habituelle pour chercher refuge dans un autre endroit à l'extérieur de son pays d'origine ou du pays dont elle a la nationalité " ${ }^{3}$. Or, l'expression "événements troublant gravement l'ordre public" couvre, entre autres, les situations économiques désastreuses en tant que cause de déplacement transfrontalier. De telles situations créent un véritable besoin de protection sans aucune distinction entre catégories sociales, socioprofessionnelles ou ethniques.

\section{Vers l'élargissement du champ de la définition des réfugiés à la persécution économique}

La Convention de Genève définit le réfugié par la persécution, mais il ne précise pas le sens exact qu'il faut donner à ce concept. Dans la représentation de sens commun, la persécution revoit le plus souvent à des actes de violence physique ou mentale, ou encore à des sanctions disproportionnées ou discriminatoires dirigées contre des personnes en raison de cinq motifs : la race, la

3 Pour plus de détails, se référer à la Convention de I'OUA (Organisation de I'Unité africaine) de 1969 régissant les aspects propres aux problèmes des réfugiés en Afrique. 
religion, I'opinion politique, la nationalité et l'appartenance à un groupe social. Bref, les actes les plus souvent dénoncés par les grandes puissances occidentales et qui se déroulèrent durant la Seconde Guerre mondiale, notamment la Shoah, les déplacements forcés et les exactions diverses telles que les exécutions, les viols, la torture, etc.

En fait, il faut rappeler que lors du processus de négociations de la Convention de Genève, deux camps se sont farouchement opposés, celui du bloc occidental et celui du bloc socialiste. Le premier défendait l'idée du réfugié comme personne persécutée. Cette définition permet de préserver la liberté politique des individus contre les gouvernements tyranniques et elle reflète ainsi I'héritage idéologique des Lumières qui promeut l'ordre libéral et démocratique, mais néglige naturellement les injustices socio-économiques (Akoka, $2013: 14$ ). Le second défendait l'idée du réfugié comme victime des violences et des inégalités socio-économiques dans une tradition communiste plus sensible aux droits collectifs. Compte tenu du contexte et surtout du pouvoir de négociation, c'est finalement la conception occidentale qui s'est imposée.

Au début du XXle siècle, le contexte international reste marqué par l'accroissement des inégalités sociales, la multiplication des crises économiques, le pillage des ressources naturelles en Afrique, l'accentuation de la dégradation des écosystèmes, etc. Compte tenu de ce contexte, la Convention de Genève pourtant fondamentale pour le droit d'asile ne semble plus vraiment adaptée. Les organismes internationaux concernés comme le Haut Commissariat des Nations unies pour les réfugiés (UNHCR) et I'Organisation internationale pour les migrations (OIM) ont d'ailleurs pris conscience de son caractère caduc. Si nous prenons par exemple le statut des milliers d'Africains subsahariens qui fuient chaque année leurs pays pour regagner l'Afrique du Nord, il parait très précaire. Ces personnes se heurtent à une sorte de vide juridique, puisqu'elles ne sont considérées ni comme immigrées (puisque "réfugiées»), ni comme réfugiées (puisque "migrantes économiques»), ni encore comme "personnes vulnérables" auxquelles les États devraient accorder protection. Certes si les persécutions, telles qu'imaginées par les rédacteurs de la Convention de Genève, sont toujours d'actualité, de nouvelles formes de persécutions sont apparues ou se sont aggravées et tout particulièrement les violences engendrées par le changement climatique et la violence économique, l'une et l'autre pouvant être liées.

S'agissant particulièrement de la violence économique, elle se manifeste dans des contextes déclencheurs ${ }^{4}$ par des comportements et des actions qui empêchent une personne d'accéder à sa liberté économique et qui contribuent sérieusement à sa fragilité sociale, à la dégradation de sa qualité de vie, voire à la menace de son existence sociale et physique. Elle englobe en particulier la restriction de la propriété privée, l'interdiction de travailler, le travail forcé, la dépossession des biens, la prolifération de l'économie de rente, l'injustice fiscale, l'exclusion sociale, la généralisation de la corruption et la saisie des salaires; c'est-à-dire des actes de privation dont la responsabilité incombe

4 Un contexte déclencheur renvoie à une situation qui favorise l'émergence ou l'aggravation des différentes formes de violence économique, en particulier la fragilité des États en Afrique et dans certains pays d'Asie et d'Amérique latine. 
essentiellement à l'État. Elle englobe également les dommages causés par la dégradation de l'environnement en raison des conditions climatiques dont la responsabilité est souvent partagée entre les pays d'origine des réfugiés et les pays d'arrivée (sécheresses, inondations, déforestations, dégradations des sols, pollutions, etc.). Des milliers de personnes sont alors tentées chaque année de quitter leurs pays uniquement afin d'échapper à de tels actes ${ }^{5}$.

Dans le cadre du système libéral, la violation des libertés économiques cadre parfaitement avec le contenu de la définition de la persécution. Certains pays comme la France ont d'ailleurs officiellement étendu la qualité de réfugié "à toute personne persécutée en raison de son action en faveur de la liberté" (article L.711-1 du CESEDA dont la formulation est inspirée de I'alinéa 4 du préambule de la Constitution de 1946). Et à l'évidence, on estime que cette liberté recouvre à la fois l'aspect politique, I'aspect civil et l'aspect économique. Mais c'est I'Office français de protection des réfugiés et des apatrides (OFPRA) qui a la compétence de se prononcer sur le lien entre la persécution et l'action en faveur de la liberté, ce qui témoigne de l'importance de la jurisprudence et donc du rôle du contexte en matière de détermination des motifs de persécution.

Dans ces conditions, il semble nécessaire de s'interroger sur les traits distinctifs du "réfugié économique" dans un contexte de mondialisation où les désastres économiques deviennent récurrents. Un "réfugié économique» renvoie alors à la situation de toute personne qui fuit seule ou en famille son pays d'origine de façon permanente ou temporaire pour échapper à une menace existentielle due aux persécutions économiques ou aux conséquences du réchauffement climatique auxquelles elle ne peut trouver de remède en restant sur place. Betts (2013) qualifie cette situation de "migration de survie". Ce terme est peut-être plus approprié pour appréhender les conditions dans lesquelles vivent la majorité des migrants économiques. II remplace tous les autres concepts utilisés habituellement par les médias et fréquemment par les chercheurs comme "migrant illégal», "migrant clandestin», "migrant sanspapiers" ou encore "étranger en situation irrégulière".

Tout migrant économique n'est pas nécessairement "économique». En effet, les personnes en situation économique favorable ou au moins tolérable, et qui quittent leurs pays juste pour améliorer davantage leurs conditions de vie, ne peuvent en aucun cas prétendre à un statut de "réfugiées économiques", car elles ont fait le choix de se déplacer de leur plein gré à la recherche de meilleures perspectives pour elles-mêmes et pour leurs familles (HCR, 2016). Le "réfugié économique" est un migrant forcé. II est confronté à des circonstances qui risquent de mettre sa vie en danger. Pourtant, certains pays riches s'opposent farouchement à cette clarification d'ordre lexical en faisant valoir l'argument habituel selon lequel "Nous ne pouvons pas ouvrir nos frontières à toute la misère du monde" pour reprendre l'expression de l'ancien premier ministre français Michel Rocard.

Nous estimons que pour être qualifiée de "réfugiée économique", une personne doit remplir les conditions suivantes:

5 À la fin 2016, et d'après les estimations de l'Agence des Nations unies pour les réfugiés (HCR), 65 millions de personnes ont été déplacées contre leur gré (HCR, 2017). 
- Avoir subi ou craindre de subir des persécutions de type économique, qui la place en situation de forte vulnérabilité ou d'extrême pauvreté. L'exemple le plus frappant est celui des millions d'Italiens qui se sont exilés aux États-Unis d'Amérique vers la fin du XIXe siècle afin de fuir la misère qui sévissait dans le pays, et qui est due en grande partie à la crise agraire de 1873. Un autre exemple assez récent concerne des milliers d'Haïtiens qui ont fui leur pays ravagé par la misère causée par les catastrophes naturelles et les problèmes de gouvernance afin de rejoindre les États-Unis ou le Canada, et plus récemment des États d'Amérique latine.

- Avoir quitté son pays, car le régime au pouvoir est incapable d'assurer sa protection contre les persécutions économiques. Un exemple éloquent est celui de milliers de Zimbabwéens qui se sont déplacés en Afrique du Sud entre 2005 et 2010 en raison de l'effondrement économique qui a frappé leur pays. Bien que le Zimbabwe recèle d'énormes richesses, son gouvernement était très affaibli par la corruption et les sanctions imposées par les pays occidentaux. II $\mathrm{n}^{\prime}$ avait pas réussi à mettre en place des politiques économiques saines, efficaces et exemptes de corruption. Les réformes agraire et monétaire qui avaient été lancées se sont soldées par des échecs, conduisant à un désastre économique et social sans précédent.

- Avoir risqué sa vie pour rejoindre un pays plus sûr. En effet, un «réfugié économique" entreprend souvent un voyage long, périlleux et clandestin. À titre d'exemple, chaque année, des milliers d'Africains subsahariens, dont des femmes parfois enceintes et des enfants, tentent de traverser des milliers de kilomètres pour rejoindre l'Europe ${ }^{6}$. Les traversées du Sahara et de la Méditerranée sont très dangereuses, car les migrants sont dans une situation de dépendance vis-à-vis des acteurs qui organisent et contrôlent les passages de manière frauduleuse et criminelle. En fait, quel que soit le type de persécution subie dans les pays d'origine (persécution politique, persécution économique, persécution religieuse, etc.), la plupart des migrants subissent des traitements cruels et dégradants comme en témoignent les propos rapportés par les rescapés de ces traversées.

- Avoir subi des persécutions visant le groupe d'appartenance. Ainsi, contrairement aux interprétations occidentales de la Convention de Genève qui retiennent le critère restrictif de la persécution personnelle, nous estimons que la persécution économique a un caractère essentiellement collectif. L'idée qui sous-tend la définition des "réfugiés économiques" est celle des violences ou des privations collectives. Ainsi, beaucoup de migrants africains subsahariens qui tentent de regagner l'Europe via le Maroc ou la Libye se plaignent des atteintes graves visant principalement les membres du groupe social qui les représentent. Naturellement, ces atteintes doivent être suffisamment intenses et étendues pour produire les effets d'une persécution collective.

- Avoir subi des persécutions économiques pour des motifs précis et limitatifs dans le temps. Les réfugiés d'Afrique de l'Ouest constituent un cas de figure exemplaire. C'est ainsi que des milliers de personnes originaires du Niger, du Mali et du Burkina Faso ont trouvé refuge hors des frontières nationales

6 D'après les chiffres publiés par l'Agence européenne de garde-frontières et de gardecôtes (Frontex), 118962 migrants, essentiellement des Nigérians, des Guinéens et des Ivoiriens, ont emprunté la route de la Méditerranée centrale menant vers I'Italie. Quant à la route de la Méditerranée occidentale menant vers l'Espagne, elle a été empruntée par 23143 migrants dont une majorité de ressortissants d'Afrique subsaharienne. 
pour échapper à l'insécurité alimentaire et aux incertitudes économiques et sociales qui en découlent (FAO, 2017). Certes, on manque de données fiables sur l'ampleur de ces déplacements transfrontaliers, mais nous estimons qu'ils sont de courte durée et de courte distance (Henry et al., $2004: 449$ ).

Remarquons, enfin, que le déplacement forcé des populations hors de leurs pays d'origine est devenu un phénomène indécomposable. En effet, les motivations qui sous-tendent les décisions de départ des personnes persécutées sont le plus fréquemment variées et parfois même complexes, car difficilement identifiables. Que cette persécution soit d'origine politique, religieuse, économique ou climatique, ses victimes se déplacent souvent côte à côte, empruntent les mêmes itinéraires, subissent les mêmes violences, recourent aux mêmes modes de transport, font appel aux services des mêmes passeurs, bénéficient éventuellement de la même assistance de la part de certaines ONG et cherchent à atteindre les mêmes destinations. Le HCR utilise un terme moins stigmatisant pour qualifier cette situation, celui de "migration mixte". Dans ces conditions, il semble dangereux du point de vue terminologique d'appliquer les termes de "réfugié politique", de "réfugié économique" ou encore de "réfugié climatique " trop hâtivement à un individu ou à un groupe d'individus sans prendre en compte la complexité du phénomène et les réalités sur le terrain.

\section{L'asile économique au Maroc : entre discours inclusif et mesures juridico-institutionnelles restrictives}

De par sa situation géographique, son ouverture et ses résultats économiques, le Maroc attire de plus en plus de "réfugiés économiques" en provenance de l'Afrique subsaharienne. La plupart d'entre eux tentent de rejoindre I'Europe, mais à défaut, ils choisissent de s'installer au Maroc. Face à l'ampleur de ce nouveau phénomène, les pouvoirs publics marocains ont choisi de tenir un discours de plus en plus humaniste et inclusif. Toutefois, le cadre juridicoinstitutionnel n'a pas beaucoup évolué et il demeure peu protecteur à l'égard de ces personnes.

\section{Le Maroc, refuge des Africains subsahariens}

Le Maroc s'est souvent présenté au fil de l'histoire comme une terre d'accueil temporaire ou permanente pour des étrangers ayant subi des persécutions politiques ou religieuses dans leurs pays d'origine. Un exemple éloquent est celui des populations juives qui ont trouvé refuge au Royaume après avoir été expulsées d'Espagne en 1492. Depuis quelques années, le Maroc accueille de plus en plus d'étrangers qui viennent $s^{\prime} y$ installer pour des raisons purement économiques. Il s'agit le plus souvent d'Africains subsahariens originaires d'Afrique de I'Ouest (Mali, Sénégal, Gambie, Guinée, Liberia, Nigeria, Ghana, etc.) et dans une moindre mesure d'Afrique centrale (République Démocratique du Congo, Cameroun, Centrafrique, etc.). Beaucoup d'entre eux vivent clandestinement au Maroc dans l'espoir de rejoindre I'Union européenne.

Bien que les chiffres officiels ne soient pas connus, le nombre de migrants originaires d'Afrique subsaharienne installés au Maroc est estimé entre 40000 et 60000 personnes. Leur déplacement est généralement assuré par une véritable économie de passage clandestin qui repose sur des réseaux de trafic et de traite 
humaine; ceux-ci se chargent d'organiser les moyens de transport, les trajets et les modalités de passage aux frontières (Wihtol de Wenden, 2005 : 11). Ces migrants empruntent des chemins de plus en plus longs et dangereux. Des dizaines d'entre eux décèdent ou sont blessés abandonnés par les passeurs durant la traversée du désert algérien.

Le principal facteur explicatif de ce flux migratoire est, semble-t-il, d'ordre purement économique. La majorité des migrants choisissent de gagner le Maroc pour fuir la misère, le chômage et la précarité économique dus à la fragilité des États ${ }^{7}$ et à la vulnérabilité des territoires accentuée par les effets de la déréglementation climatique. Cette mobilité est naturellement facilitée par l'accès rapide à l'information et la réduction des coûts de transports informels. Le récit des migrants ayant réussi à traverser le désert algérien constitue également un facteur qui incite de nouveaux migrants à tenter leur chance.

Certes, le Maroc n'offre pas assez d'opportunités en matière d'emplois et de bien-être économique, mais il reste très prisé par les migrants subsahariens. Sa proximité de I'Union européenne, la présence de deux enclaves encore occupées par l'Espagne sur sa côte méditerranéenne (Ceuta et Melilla), sa stabilité politique et son niveau de développement relativement avancé par rapport à certains pays africains sont autant de facteurs qui expliquent cette préférence. À cela s'ajoute la mémoire des formes anciennes de mobilités entre le Maroc et I'Afrique subsaharienne (la diffusion de I'Islam, le commerce des biens et le commerce des esclaves).

Une fois au Maroc, ces migrants se retrouvent piégés dans une situation de précarité économique et sociale. Faute de pouvoir atteindre l'Europe, beaucoup d'entre eux vivent clandestinement dans les quartiers populaires des grandes villes du Royaume, mais aussi dans des camps de fortune dans les forêts mitoyennes aux deux enclaves espagnoles de Ceuta et Melilla (le camp de Gourougou près de Nador et le camp de Belyounech près de Tétouan). Traditionnellement pays de transit, le pays se transforme petit à petit en terre d'accueil. Des centaines, voire des milliers, d'Africains subsahariens se sont discrètement installés à Casablanca, mais aussi àTanger, Rabat, Oujda, Tétouane, Nador, etc. Certains travaillent au noir dans des emplois subalternes et précaires qui favorisent les pratiques d'exploitation comme dans les secteurs de l'agriculture et des travaux domestiques; d'autres s'adonnent à la mendicité près des mosquées et des feux de signalisation afin d'assurer leur survie. Pourtant, un grand nombre d'entre-eux ont un niveau de formation élevé. En effet, d'après une enquête réalisée par le Haut Commissariat marocain au plan (HCP), 75,7 \% des immigrants installés au Maroc sont titulaires d'un diplôme (HCP, $2017: 23$ ).

\footnotetext{
7 D’après I'OCDE (Organisation de Coopération et de Développement Économiques), " un Etat fragile a une faible capacité à exercer des fonctions de base de la gouvernance, et n'a pas la capacité de développer des relations mutuellement constructives avec la société. Les Etats fragiles sont également plus vulnérables aux chocs internes ou externes tels que la crise économique ou les catastrophes naturelles. En revanche, les états plus résistants présentent la capacité et la légitimité pour gouverner une population et son territoire" (OECD, 2012).
} 


\section{Un discours de plus en plus humaniste et inclusif}

Depuis 2013, le discours migratoire prôné par les autorités marocaines a radicalement changé. Le discours imprégné de méfiance et d'hostilité à l'égard des immigrés, en particulier les Africains subsahariens, a rapidement laissé la place à un discours de plus en plus humaniste et inclusif. Ce changement d'attitude se manifeste à travers cinq voies majeures.

En premier lieu, des directives royales ont été données au gouvernement afin d'élaborer "une nouvelle politique globale relative aux questions d'immigration et d'asile, suivant une approche humanitaire conforme aux engagements internationaux du Maroc et respectueuse des droits des immigrés " (extrait du discours royal du 6 novembre 2013). Le souverain s'est engagé «à œuvrer pour remédier aux causes réelles de l'immigration en la reliant au développement et en adoptant une approche humanitaire et solidaire, protégeant les droits des immigrés et préservant leur dignité " (extrait du discours du Roi Mohamed VI du 20 août 2016).

En deuxième lieu, le Maroc a tenu à réaffirmer le caractère indépendant et souverain de sa nouvelle orientation en matière de politique migratoire. II a exprimé son refus de se soumettre aux pressions économiques et aux choix sécuritaires de l'Union européenne, estimant que la question migratoire devra être abordée dans le cadre d'une approche intégrée et globale.

En troisième lieu, la qualification officielle donnée aux étrangers en situation illégale sur le territoire marocain a subi une transformation d'ordre sémantique. Des expressions cristallisant les stéréotypes comme "les immigrés clandestins", "les étrangers", les "immigrants illégaux", les " harraga» (ceux qui "brûlent les frontières" en risquant leur vie) ont été rapidement remplacées par l'expression "migrants en situation administrative irrégulière". Ce changement reflète l'importance que l'État marocain commence à accorder au statut juridique de ces personnes et témoigne en même temps de sa volonté de régulariser leur situation conformément aux dispositions réglementaires en vigueur.

En quatrième lieu, et dans le cadre de sa nouvelle politique extérieure africaine marquée, entre autres, par son retour au sein de I'Union africaine, son dynamisme au sein de la communauté des États sahélo-sahariens (CEN-SAD) et sa demande d'adhésion à la Communauté économique des États de l'Afrique de l'Ouest (CEDEAO), le Maroc s'est lancé dans une opération de séduction grâce à la question migratoire en Afrique. Cette opération, qui vise également à renforcer la position du Royaume quant à son intégrité territoriale, a pris la forme d'un engagement formel quant à la mise en œuvre d'une coopération agissante et mutuellement avantageuse avec cet espace africain. Parmi les promesses avancées, nous pouvons citer la création d'un Observatoire africain sur la migration à Rabat, le rejet de la proposition de I'Union européenne d'accueillir des centres de rétention destinés essentiellement aux migrants originaires de l'Afrique subsaharienne et la disposition du Maroc à partager son expérience et son expertise dans le domaine de la gestion des flux migratoires avec ses partenaires africains. 
En cinquième lieu, un effort de communication et de sensibilisation a été mené autour de la nouvelle politique migratoire prônée par le Maroc. La compagne lancée dans ce sens se veut plus large, plus chaleureuse et plus instructive. Celle-ci vise quatre objectifs : la diffusion de la vision, des objectifs et des actions définis dans le cadre de la stratégie en question; la sensibilisation aux enjeux de l'immigration pour le Maroc ; la mobilisation de l'ensemble des parties prenantes autour du processus de mise en œuvre de cette stratégie; et la communication sur l'état d'avancement de la mise en œuvre de la stratégie, les acquis et les résultats.

Sur le plan pratique, et outre l'implication d'intervenants et de consultants de renommée (artistes, politologues, journalistes, hommes politiques, etc.) lors des émissions radio et télévisées, plusieurs rencontres ont été organisées par les pouvoirs publics pour présenter aux différentes parties concernées (corps diplomatique accrédité à Rabat, société civile, médias, institutions onusiennes, etc.) les principes généraux et les modalités de régularisation des migrants en situation irrégulière.

La plupart des médias marocains ont largement répercutés les positions officielles en commençant à énoncer un discours plus équilibré et plus humaniste. Les dérapages parfois racistes que véhiculaient certains médias écrits et électroniques quant au phénomène de l'immigration clandestine laissent progressivement place à une couverture médiatique plus solidaire et assez favorable à la cause des étrangers en situation irrégulière ${ }^{8}$. Depuis 2013, date du lancement de la nouvelle stratégie migratoire, les médias se sont largement mobilisés pour combattre les images stéréotypées et le racisme culturel présents au sein d'une partie de la société marocaine.

\section{Un cadre juridique et institutionnel peu protecteur}

En dépit de sa nouvelle position comme terre d'accueil ou de transit pour les "migrants de survie" issus de l'Afrique subsaharienne, et à l'instar du reste de la communauté internationale, le Maroc ne reconnait pas le statut de "réfugié économique". Seules les personnes persécutées pour des raisons non économiques et non climatiques peuvent prétendre bénéficier du droit d'asile.

Depuis son indépendance en 1956, le Maroc s'est progressivement engagé dans un processus de construction d'un arsenal juridique et institutionnel régissant le droit d'asile et d'immigration. Cette construction a porté autant sur des instruments internationaux que nationaux. Au niveau international, le Maroc a ratifié dès les premières années de son indépendance la plupart des Conventions relatives à la protection des réfugiés et demandeurs d'asile. Il s'agit en particulier de la Convention de Genève de 1951, du Protocole de 1967 relatif au statut des réfugiés et de la Convention de I'OUA régissant les aspects propres aux problèmes des réfugiés en Afrique. En ratifiant ces conventions, le Maroc

8 Deux faits négatifs ont marqué la couverture médiatique des immigrés en situation irrégulière sur le territoire marocain à la veille du lancement de la nouvelle politique migratoire. Ainsi, en 2005, I'hebdomadaire Achamal a comparé les immigrés subsahariens à des "criquets noirs" envahissant le Nord du Maroc. De même, en 2012, un autre hebdomadaire, Maroc Hebdo International, a qualifié la présence de Subsahariens sur le sol marocain de "péril noir". 
songeait essentiellement à ses propres migrants installés en Europe (Alami M'Chichi, 2005 : 29). En fait, durant les premières années de l'indépendance, des milliers de Marocains se sont progressivement installés en Europe de l'Ouest, principalement en France, en Allemagne, aux Pays-Bas et en Belgique ${ }^{9}$. II s'agit le plus souvent de travailleurs migrants engagés pour combler le manque de main-d'œuvre dans un contexte économique européen de forte croissance.

Le Maroc a également ratifié d'autres Conventions qui ont des implications directes ou indirectes sur la situation des réfugiés. Citons à titre d'exemples: le Pacte international relatif aux Droits civils et politiques; le Pacte international relatif aux droits économiques, sociaux et culturels; la Convention internationale sur l'élimination de toutes les formes de discrimination raciale; la Convention contre la torture et autres peines ou traitements cruels inhumains ou dégradants; la Convention relative aux droits de l'enfant; la Convention internationale sur la protection des droits de tous les travailleurs migrants et des membres de leur famille.

Au niveau national, le Maroc s'est doté depuis 1957 d'une législation relative au droit d'asile (le Décret du 29 août 1957 fixant les modalités d'application de la Convention relative au statut des réfugiés). Deux institutions furent créées en vertu de ce texte : le bureau des Réfugiés et Apatrides (BRA) rattaché au ministère des Affaires étrangères et la Commission de recours composé du ministre de la Justice (ou de son représentant), le ministre des Affaires étrangères (ou de son représentant) et le représentant du HCR auprès du Maroc.

En 2003, le Maroc a promulgué la loi $n^{\circ} 02-03$ relative à l'entrée et au séjour des étrangers, à l'émigration et l'immigration irrégulières. Cette loi est venue encadrer et restreindre pour la première fois l'entrée et le séjour des étrangers au Maroc. Sa promulgation intervient dans un contexte humainement peu propice. En effet, suite aux attentats de Casablanca du 16 mai 2003, une loi antiterroriste très répressive a été adoptée. Un certain amalgame a été réalisé entre migrants et terroristes, puisque l'immigration illégale est élevée au rang de délit passible de poursuites pénales. La même année, le Maroc et I'Union européenne ont entamé la négociation d'un accord de réadmission qui vise à faciliter le renvoi forcé vers le pays d'origine ou de transit des migrants arrêtés en situation irrégulière sur le territoire d'un État européen d'accueil. S'appuyant sur le principe de la conditionnalité (signature d'un accord de réadmission en contrepartie d'un accord de facilitation de visas pour les ressortissants marocains), la partie européenne a voulu faire pression sur le Maroc pour adhérer à sa politique sécuritaire basée, entre autres, sur l'externalisation des contrôles migratoires. Parallèlement, et au cours de la même année, le Maroc a lancé les premières opérations de rapatriement forcé des exilés d'Afrique subsaharienne vers leurs pays d'origine, opérations largement critiquées par les médias et la société civile, mais saluées par les partenaires européens, en particulier l'Espagne.

La loi $n^{\circ}$ 02-03 insiste sur la nécessité de respecter les engagements internationaux souscrits par le Maroc en matière de droits de l'Homme et de droits des

9 Suite aux Accords bilatéraux de recrutement de main-d'œuvre signés avec la France en 1963, I'Allemagne en 1963, la Belgique en 1964 et les Pays-Bas en 1969, le nombre de Marocains résidant en Europe a sensiblement augmenté. II est passé de 30000 en 1965 à 137000 en 1968 avant d'atteindre 394000 en 1975 (Alaoui, 2013 : 9). 
réfugiés. Selon son article 17, la carte de résidence est délivrée, entre autres, à l'étranger qui a obtenu le statut de réfugié en application du décret du 29 août 1957, fixant les modalités d'application de la Convention relative au statut des réfugiés, ainsi qu'à son conjoint et à ses enfants mineurs ${ }^{10}$. De même, en vertu de l'article 29 de ladite loi, aucune expulsion ne peut être prononcée à l'encontre des femmes étrangères enceintes et des mineurs étrangers. Enfin, aucun étranger ne peut être éloigné à destination d'un pays s'il établit que sa vie et/ou sa liberté y sont menacées ou qu'il y est exposé à des traitements inhumains, cruels et dégradants.

Mais en dépit de ces avancées modestes, la loi en question semble être liberticide sur certains points. D'une part, elle est très répressive à l'égard des "refugiés économiques", et ce, à travers le renforcement du contrôle des immigrés subsahariens arrivant au Maroc avec possibilité d'intervention policière, de mise en rétention, de refoulement et même de remise en cause des papiers relatifs à l'octroi ou à la demande d'asile. D'autre part, cette loi est muette sur des questions comme le regroupement familial, les droits de défense en phase d'enquêtes préliminaires, l'égalité de genre, l'information relative aux droits des migrants et aux modalités de notification des décisions de reconduite à la frontière.

Quoi qu'il en soit, cette loi est devenue caduque depuis l'entrée en vigueur de la nouvelle constitution de 2011, et surtout suite à l'annonce de la nouvelle stratégie migratoire initiée en septembre 2013 (Stratégie nationale d'Immigration et d'Asile). Trois projets de loi ont été alors élaborés dans la perspective de l'abrogation de celle de 2003 : un projet de loi sur le droit d'asile, un projet de loi relatif au séjour des étrangers et un projet de loi portant sur la traite des êtres humains. Mais jusqu'à présent, seule la loi 27-14 sur la traite des êtres humains fut adoptée. Elle vise essentiellement à harmoniser la législation du Royaume avec les conventions internationales. Les deux autres projets de loi tardent à être présentés au Parlement. Officiellement, ce retard a été justifié par le souci d'approfondir l'étude des textes eu égard à leur importance, mais la réalité est que le processus de négociation avec les parties concernées, en particulier la société civile, n'a pas abouti. En fait, sur un dossier aussi sensible que celui du droit d'asile, il est souvent difficile de trouver un compromis entre les impératifs sécuritaires de l'État et les aspirations humanitaires des ONG et des institutions onusiennes concernées (HCR et OIM). À cela s'ajoute le manque d'expertise et d'audace de la part des législateurs chargés de l'élaboration des lois. Si la première limite (le manque d'expertise) trouve son origine dans le faible niveau de connaissances et de compétences, la seconde (le manque d'audace) s'explique plutôt par le désir des législateurs de préserver leurs intérêts politiques.

Sur un autre registre, la réforme constitutionnelle de 2011 a affirmé les racines multiculturelles africaines du Maroc et instauré en même temps une égalité de traitement entre les Marocains et les étrangers. Désormais, la présence des étrangers sur le territoire marocain a une valeur constitutionnelle. Ainsi, l'article 30 de la Constitution stipule que "les étrangers jouissent des libertés

10 Voir la loi $n^{\circ} 02-03$ relative à l'entrée et au séjour des étrangers au Royaume du Maroc, à l'émigration et à l'immigration irrégulière, promulguée par le Dahir n 1-03-196 du 11 novembre 2003. 
fondamentales reconnues aux citoyennes et citoyens marocains, conformément à la loi. Ceux d'entre eux qui résident au Maroc peuvent participer aux élections locales en vertu de la loi, de l'application de conventions internationales ou de pratiques de réciprocité ". Cet article témoigne de la volonté des pouvoirs publics marocains de faire face aux changements structurels que connaît le phénomène migratoire. Toutefois, son contenu demeure très vague et surtout muet quant au sort qui sera réservé aux "réfugiés économiques». En effet, puisque les juristes et les politiciens ont souvent une interprétation très stricte de la Constitution, les migrants économiquement persécutés sont exclus de toute possibilité d'accès au statut de réfugié. Or, cette exclusion est largement infondée si l'on considère la dimension humanitaire dans le cadre d'une approche migratoire cohérente, équilibrée et durable.

Parallèlement à ce dispositif $d^{\prime}$ 'ordre juridique, le Maroc a initié une réforme institutionnelle dynamique et multifacette. Ainsi, en 2004, une direction de la Migration et de la Surveillance des frontières fut créée. Rattachée au ministère de I'Intérieur, cette direction a pour principale mission l'opérationnalisation de la stratégie nationale en matière de lutte contre les réseaux de trafic des êtres humains et la surveillance des frontières. Il a été également procédé à la création d'un Observatoire de la migration. Celui-ci est notamment chargé de centraliser les informations liées à la migration, de mener des recherches portant sur les tendances des flux migratoires et de proposer aux pouvoirs publics des mesures concrètes sur les questions se rapportant à ce sujet. De plus depuis le début des années 2000, la coopération entre le Maroc et les institutions onusiennes chargées des questions migratoires s'est nettement améliorée. Cette coopération s'est concrétisée par la signature de deux accords, I'un avec I'Organisation internationale pour les migrations (signé en 2005) et l'autre avec le Haut Commissariat des Nations unies pour les réfugiés (signé en 2007). Ces deux accords précisent les dispositions relatives à l'ouverture de représentations qui permettront à ces institutions de s'acquitter de leurs mandats sur le territoire marocain.

Le Maroc a également adopté en décembre 2014 un projet de loi relatif à la stratégie nationale de la migration et de l'asile. Ce projet vise quatre principaux objectifs (Bernoussi et Benkeroum, 2017 : 429) : la gestion des flux migratoires dans le respect des droits de l'Homme, la mise en place d'un cadre institutionnel adapté, la facilitation de l'intégration des immigrés réguliers, et la mise à niveau du cadre réglementaire. Dans le cadre de la mise en œuvre de cette nouvelle stratégie, le Maroc a donné son feu vert à l'ouverture d'un bureau des Réfugiés et des Apatrides à Rabat. Ce bureau a été chargé dans un premier temps de valider les dossiers des réfugiés antérieurement reconnus par le HCR Maroc. Dans un deuxième temps, il s'est attelé à l'assistance aux demandeurs d'asile et à la mise en œuvre de la procédure de la détermination du statut de réfugié. Parallèlement, il a été procédé à la mise en place d'une Commission nationale de suivi et de recours. Constituée de représentants des principaux départements ministériels concernés (les Affaires étrangères, I'Intérieur, la Justice, l'Emploi et les Droits de l'Homme) et d'un représentant de la délégation du HCR à Rabat, cette Commission a pour principale mission de veiller à ce que l'opération de l'examen des demandes d'asile se déroule selon une approche souple et efficiente, et ce, à travers l'adoption du système de guichet unique. Elle a aussi pour but de formuler des propositions et des avis dans le domaine de l'insertion des 
groupes de migrants régularisés et de sensibiliser la société marocaine dans son ensemble à la question de la migration et de l'asile. Bien qu'il soit encore trop tôt pour se prononcer sur les résultats de ce dispositif institutionnel, il semble qu'il n'ait pas encore produit tous les effets escomptés. Les procédures d'exécution sont jugées trop lentes et complexes. De même, le dispositif en question reste muet quant aux violences et aux actes racistes dont sont victimes certains "réfugiés économiques» originaires d'Afrique subsaharienne.

\section{La gestion de la question des « réfugiés économiques " au Maroc}

L'arsenal juridique et institutionnel mis en place par le Maroc pour encadrer le droit des immigrés en général est assez riche. Toutefois, son effectivité est souvent contestée, en particulier concernant la protection et l'inclusion des "réfugiés économiques".

\section{Une stratégie hésitante et sélective}

Comme dans d'autres pays confrontés aux flux migratoires, la question des demandeurs d'asile et des réfugiés au Maroc a pendant longtemps souffert d'un certain immobilisme. Cela s'explique essentiellement par l'afflux relativement faible d'immigrés durant les années 1970 et1980 et le fait que les pouvoirs publics aient été focalisés sur d'autres sujets pesants en particulier le parachèvement de l'intégrité territoriale. À cela s'ajoute un sentiment d'indifférence de I'opinion publique marocaine à l'égard de cette question.

Avec les arrivées significatives des Africains subsahariens à partir des années 1990, la politique migratoire marocaine a subi des bouleversements. D'une politique inactive, le pays est passé à une politique réactive, mais globalement hésitante et sélective. Par crainte de se transformer en terre d'arrivée d'Africains subsahariens, le Maroc a adopté une attitude hybride, tantôt orientée vers la fermeté (arrestations, détentions provisoires, refoulements aux frontières, déplacements, etc.), tantôt vers davantage de souplesse (régularisation ciblée et ponctuelle, assistance aux réfugiés, communication positive, etc.). Le but était d'exercer un effet dissuasif sur les nouveaux arrivants, tout en faisant monter les enchères vis-à-vis de I'Union européenne, qui compte sur l'aide du Maroc afin de ralentir l'afflux des migrants subsahariens, composés essentiellement de "réfugiés économiques" vers la rive nord de la méditerranée.

Actuellement, les procédures d'examen et de traitement des demandes d'asile ou de régularisation sont jugées encore trop sélectives, trop longues et trop complexes comparativement à celles mises en place dans les pays développés. Elles sont souvent laissées à la discrétion des fonctionnaires du ministère de I'Intérieur. Or la compétence et l'expertise de ces derniers ne sont pas encore complètement acquises. À cela s'ajoute une bureaucratie qui pèse lourdement sur l'administration marocaine, avec tout ce que cela induit en termes d'abus de pouvoir, de dégradation de la qualité du service et de développement des pratiques clientélistes et corruptrices. 
Alors que les données officielles sur les réfugiés politiques existent et sont régulièrement publiées (7 138 réfugiés demandeurs d'asile au 31 mars 2018) ${ }^{11}$, celles concernant les arrivants que nous avons qualifiés de "réfugiés économiques" font défaut. Certes, le Maroc a régularisé la situation de quelque 25000 migrants depuis 2014, mais ces personnes étaient déjà intégrées de fait. En effet, $d$ 'après la circulaire régissant la première opération exceptionnelle de régularisation de la situation de séjour des étrangers, publiée en décembre 2013, les critères d'admission reposent sur le degré d'intégration au Maroc, les liens familiaux et les cas humanitaires. Au total, six catégories d'étrangers ont bénéficié de cette régularisation : les étrangers conjoints de ressortissants marocains, les étrangers conjoints $d$ 'autres étrangers en résidence régulière au Maroc, les enfants issus des deux cas susvisés, les étrangers disposant de contrats de travail effectifs, les étrangers justifiant de cinq ans de résidence continue au Maroc et enfin les étrangers atteints de maladies graves. Or au regard d'une situation conforme à la jurisprudence issue du droit comparé et des pratiques internationales, ces personnes étaient déjà censées bénéficier d'une carte de séjour sans la moindre réserve et peut-être aussi sans avoir besoin de médiatiser fortement cette initiative humanitaire.

L'approche préconisée par le Maroc a, semble-t-il, un caractère exclusif, car ponctuelle et axée sur des groupes spécifiques de migrants. D'abord, la régularisation a été limitée dans le temps. Le délai prévu pour la première opération par exemple s'étale sur une année. Ensuite, la régularisation a profité essentiellement aux femmes puisque tous leurs dossiers (5 000 dossiers) ont été acceptés; la commission de recours a pris en compte la vulnérabilité spécifique de cette catégorie de migrants. Enfin, l'élément politique est fortement présent lors de la régularisation de la situation des migrants. Ce sont généralement les migrants originaires des pays entretenant de bonnes relations avec le Maroc, en particulier le Sénégal et la Côte d'Ivoire, qui ont bénéficié de cette opération de régularisation.

Pour les arrivants que nous qualifions de "réfugiés économiques", la situation est préoccupante. Ils sont en effet aujourd'hui dans une véritable impasse, car ils ne sont ni régularisés ni expulsés en respectant les procédures. Leurs conditions de vie sont extrêmement précaires et difficiles. Ils sont socialement exclus et parfois objets d'actes de racisme et de discrimination (insultes dans les lieux publics, agressions symboliques, injures, discriminations dans I'accès au logement et au travail décent, etc.). Pour assurer leur survie, beaucoup d'entre eux sont contraints de recourir à la mendicité et à l'exercice de certaines activités informelles faiblement rémunérées.

Signalons enfin qu'une deuxième compagne de régularisation a été lancée en décembre 2016. Quelque 26000 demandes ont été déposées dont $33 \%$ par des femmes et $9 \%$ par des mineurs, mais les critères d'éligibilité n'ont pas changé. Le traitement des dossiers est conduit selon les mêmes conditions sélectives exigées lors de la première opération (degré d'intégration au Maroc,

11 Ce chiffre comprend 3763 réfugiés et 1376 demandeurs d'asile. Les hommes représentent $58,5 \%$ de l'effectif total contre $41,5 \%$ pour les femmes. La majorité est originaire de Syrie avec 3062 réfugiés, du Cameroun avec 701 réfugiés, de la Guinée avec 612 réfugiés et duYémen avec 556 réfugiés (HCR, 2016). 
liens familiaux et cas humanitaires). Or de telles conditions sont difficilement remplies par les "réfugiés économiques", ce qui risque de dissuader des milliers d'entre eux d'essayer d'être régularisés. En même temps, le Bureau des réfugiés et des apatrides au Maroc a suspendu ses services en mars 2017. Les audiences n'ont plus lieu. Officiellement, aucune explication n'a été fournie pour justifier cette suspension, mais il semble que des désaccords soient apparus entre les autorités marocaines et le HCR au sujet de l'identification des demandeurs d'asile. Par conséquent, plusieurs centaines de personnes reconnues comme réfugiées par le HCR se retrouvent dans une situation d'incertitude. Cette nouvelle réalité traduit encore une fois le caractère hésitant et parfois paradoxal de la politique migratoire marocaine. D'un côté, un effort de sensibilisation et de communication agressive a été déployé, de l'autre, le processus d'implémentation des lois et des directives demeure très lent et compliqué.

\section{Une stratégie réactive et politiquement opportuniste}

Confronté à la pression migratoire, le Maroc n'a jamais réussi à construire une véritable politique proactive. Plutôt que d'anticiper l'évolution des flux migratoires et proposer des solutions pragmatiques et flexibles, les pouvoirs publics marocains préfèrent souvent attendre le contexte politiquement approprié pour agir. La première compagne de régularisation des sans-papiers par exemple, celle de 2014, a été déclenchée à la suite de la publication d'un rapport alarmant sur la situation des migrants et des réfugiés au Maroc (CNDH, 2013). Elle s'inscrit également dans un contexte d'ouverture politique, caractérisé par l'adoption d'une nouvelle Constitution et la création de l'instance équité et réconciliation qui a pour but de réconcilier les Marocains avec leur passé récent (les années de plomb sous le règne du Roi Hassan II) et remédier aux violations des droits de I'Homme. Quant à la deuxième compagne de régularisation lancée en décembre 2016, elle s'inscrit dans un contexte assez particulier caractérisé par une forte mobilisation diplomatico-économique menée par le Royaume pour réintégrer I'Union africaine après trois décennies d'absence. Le Maroc, qui a désormais un regard stratégique sur ses relations avec l'Afrique subsaharienne, cherche également à se différencier de son voisin et "ennemie politique", l'Algérie, dans le traitement réservé aux migrants africains, et alors que l'Algérie durcit ses positions migratoires en reconduisant systématiquement aux frontières les migrants subsahariens qui arrivent sur son territoire. D'après les estimations de I'OIM, les autorités algériennes auraient abandonné plus de 13000 migrants dans le désert du Sahara entre mai 2017 et juin 2018 (Hinnant, 2018).

Les deux compagnes de régularisation constituent également une réponse aux critiques, parfois virulentes, d'ONG internationales (Fédération internationale des Droits de I'Homme, Médecins sans frontières, Terre des Hommes, Amnesty International, etc.). Celles-ci dénoncent surtout l'usage excessif de la violence à l'égard des immigrés qui tentent de rejoindre les enclaves occupées de Ceuta et Melilla, de même que les conditions de précarité dans lesquelles vivent ceux qui finissent par élire domicile au Maroc (Qassemy, 2014). Par ailleurs, et malgré le discours officiel marqué par le refus d'endosser le rôle de gendarme migratoire de I'Union européenne, le Maroc continue de prêter main-forte à I'Espagne dans la traque des migrants non désirés. Des milliers de soldats ont été déployés au nord du Royaume essentiellement pour empêcher 
les immigrés subsahariens d'atteindre l'Europe ou de s'infiltrer dans les enclaves de Ceuta et Melilla. D'ailleurs, la loi $n^{\circ} 02-03$ relative à l'entrée et au séjour des étrangers au Maroc, à l'émigration et l'immigration irrégulière s'inscrit parfaitement dans cette logique de protection des frontières européennes. Ce fossé entre le discours politique et la réalité s'explique essentiellement par des considérations géostratégiques telles que les avantages commerciaux procurés par le statut avancé de 2008, le souci sécuritaire lié au risque d'attaques terroristes et le respect de l'intégrité territoriale du Royaume. Certes, l'Accord d'association signé entre les deux parties en 1996 n'inclut pas expressément une telle clause, mais le lancement de la politique de voisinage a introduit la question migratoire dans I'agenda euro-marocain et une Déclaration allant dans ce sens a été signée en 2013. Celle-ci stipule, entre autres, que le Maroc et l'Union européenne «vont coopérer pour mieux lutter contre les réseaux de trafic de migrants et de traite d'êtres humains et venir en aide aux victimes. Ils travailleront de manière étroite pour assister le Maroc à mettre en place un système national d'asile et de protection internationale ${ }^{12}$.

Si le Maroc a déployé d'énormes efforts en matière de sécurisation de sa frontière méditerranéenne, il a toujours rejeté le projet d'Accord de réadmission proposé par l'Union européenne, et ce, malgré sa mise sur la table des négociations depuis 2003. Ce type de mécanisme obligerait en effet le Maroc à accueillir tous les Africains subsahariens ayant transité par son territoire avant d'atteindre clandestinement le sol d'un pays membre de l'Union européenne. Or une telle action risquerait d'affecter la crédibilité de la nouvelle politique africaine du Maroc qui se veut offensive et multidimensionnelle.

De plus, la conclusion d'un Accord de réadmission des ressortissants des pays tiers pousserait éventuellement le Maroc à mettre en place des centres d'accueil pour les Africains subsahariens réadmis en attendant leur expulsion vers leurs pays d'origine, chose que les responsables marocains ont toujours rejetée en faisant valoir le principe de la dignité humaine. Cette situation pourrait en effet nuire à l'image du Royaume comme défenseur des droits de l'Homme et protecteur des droits fondamentaux des migrants, fondement même de sa nouvelle politique migratoire. À cela s'ajoute I'opposition farouchement exprimée par les associations de défense des droits des immigrés, qui voient dans ces centres le symbole même d'une politique migratoire inefficace et dangereuse, comme en témoigne l'expérience espagnole dans ce domaine.

Certes, le Maroc a déjà ratifié plusieurs accords bilatéraux de réadmission de ses émigrés ainsi que des étrangers ayant transité par son territoire pour se rendre dans I'Union européenne, mais le degré d'application de ces accords reste très faible, voire insignifiant ${ }^{13}$. La raison tient essentiellement au flou

12Pour plus de détails, voir la Déclaration conjointe établissant un partenariat de mobilité entre le Royaume du Maroc, I'Union européenne et ses États membres signée le 7 juin 2013 au Luxembourg, document disponible sur le site : http://www.sharaka.ma/assets/ Uploads/Mobilite-Declaration-UE-Maroc-Juin-2013.pdf

13 Le Maroc a signé des accords de réadmission avec ses principaux partenaires européens, en particulier: I'Allemagne (1er juin 1998), la France (1993 et 2001), le Portugal (7 septembre 1999), I'Italie (27 juillet 1998 et son protocole additionnel du 18 juin 1999), I'Espagne (13 février 1992 et 23 décembre 2003), la Belgique (22 avril 2016), les Pays-Bas (22 avril 1993) et le Royaume-Uni (24 septembre 2011). 
juridique qui entoure les critères de réadmission, en particulier pour les ressortissants des pays tiers. Chaque partie contractante est libre d'apprécier les circonstances dans lesquelles ces personnes sont entrées illégalement dans le pays européen. Le Maroc soulève souvent la question des éléments de preuves quant au passage par son territoire des migrants africains subsahariens avant leur arrivée en Europe. Et même en présence de preuves irréfutables, la collaboration des autorités marocaines n'est pas toujours au rendez-vous en raison du caractère politiquement sensible de l'opération de réadmission des ressortissants des pays de l'Afrique subsaharienne. Cela est d'autant plus compréhensible que ces accords s'attaquent le plus souvent aux effets et non aux véritables causes du phénomène migratoire. En conséquence, des accords de ce type n'ont qu'une valeur symbolique.

Signalons au passage que malgré l'absence d'accord officiel entre le Maroc et I'Union européenne portant exclusivement sur les questions migratoires, le niveau de coopération informelle entre les deux parties a toujours été à un niveau élevé. Cela se manifeste à travers l'échange d'informations, le transfert d'équipements techniques et du savoir-faire vers les gardes-frontières marocains et le renforcement des capacités des services d'immigration (Cassarino et Lavenex, $2012: 312$ ).

Au vu de ces éléments, il semble que malgré la combinaison d'opérations de séduction et de pression de la part de I'Union européenne, le Maroc n'ait jamais totalement adhéré à la politique d'externalisation migratoire voulue par Bruxelles. Sa stratégie consiste à faire perdurer les négociations le plus longtemps possible. En fait, dans ce type de situation, les discussions ne servent pas forcément à parvenir à un accord quelconque, mais à manœuvrer en fonction des événements et de l'évolution des contraintes géopolitiques tout en cherchant à préserver un certain équilibre entre les relations historiques avec l'Europe et le désir du Maroc de se positionner sur le très convoité marché africain.

\section{Une stratégie participative et sociétale}

Bien que la gestion des flux migratoires soit un droit régalien, les pouvoirs publics marocains ont adopté une approche participative impliquant toutes les parties prenantes : opérateurs publics et privés, société civile, média, syndicats et organisations internationales. Cela témoigne de la volonté du Maroc à lever les préjugés qui entourent la question migratoire et à construire une vision partagée en matière de définition d'une politique $d^{\prime}$ 'asile et d'accueil des "réfugiés économiques". Cela témoigne également de l'incapacité du Maroc à assumer seul la responsabilité de la prise en charge des populations en quête d'un refuge à l'intérieur de ses frontières.

Concernant particulièrement la société civile, elle est devenue aujourd'hui une partenaire incontournable en matière de gestion des flux migratoires. Ses actions sont de plus en plus visibles et appréciées par tous. Outre les compagnes de sensibilisation et d'assistance humanitaire, le tissu associatif marocain est très actif dans le domaine des droits humains, du plaidoyer et d'accompagnement. D'une part, il assure le suivi de la situation juridique des réfugiés et de la législation sur la migration au Maroc. II œuvre également pour 
le respect de la dignité et de l'égalité de traitement des "réfugiés économiques", mais aussi contre toutes les formes de discrimination et de racisme. D'autre part, le tissu associatif marocain est de plus en plus actif dans le domaine de l'intégration économique des réfugiés et des migrants. Il accompagne techniquement et financièrement ceux d'entre eux qui souhaitent développer leurs propres activités professionnelles dans le cadre de l'économie solidaire.

La société civile marocaine a eu le mérite de créer un véritable espace de débat et de réflexion sur les différents aspects de la question migratoire. Elle interpelle systématiquement les pouvoirs publics sur les problèmes d'asile et propose des alternatives pour améliorer les conditions de vie des réfugiés qu'ils soient politiques ou économiques. Les recommandations formulées ont souvent trait à la protection des réfugiés et au respect de leurs droits, à leur accès à un travail décent, à leur accès au logement, à l'accès de leurs enfants à l'école, etc. De telles recommandations sont généralement portées à la connaissance des pouvoirs publics à travers des circuits institutionnels. En effet, depuis quelques années, la société civile marocaine s'est impliquée dans l'élaboration et la mise en œuvre des stratégies migratoires à l'échelle nationale, mais aussi dans les concertations relatives à l'élaboration des projets de loi sur la traite des êtres humains et sur le droit d'asile. Certaines associations de plaidoyer œuvrant dans le domaine migratoire vont même jusqu'à la production des connaissances sur le sujet, et ce à travers l'organisation de manifestations scientifiques (workshops, colloques, journées de formation, etc.), la réalisation d'études de terrain et la publication de rapports. Cette production est d'autant plus importante qu'elle s'effectue généralement en collaboration avec des structures de recherche nationales ou internationales. Parmi les associations actives dans ce sens, citons le groupe antiraciste de défense et d'accompagnement des étrangers et migrants (GADEM), I'Association des amis et familles des victimes de l'immigration clandestine (AFVIC) et le conseil des migrants subsahariens au Maroc (CMSM).

\section{Conclusion}

Au terme de cette réflexion, il semble que le qualificatif de "réfugiés économiques" n'est que l'une des nombreuses illustrations de la mondialisation néolibérale et l'échec du modèle de développement qui s'y rattache et qui a été transposé aux pays du Sud sans tenir compte des contraintes locales. Ainsi, avec la multiplication des crises, associée aux erreurs de politiques économiques, à la fragilité des États et à la dégradation des conditions climatiques, les désastres économiques se sont succédé, poussant un nombre élevé de personnes en situation de vulnérabilité à quitter leur domicile pour se réfugier dans un autre pays. Ce sont ces personnes que nous avons qualifiées ici de "réfugiés économiques".

Le Maroc, de par sa position charnière avec l'Europe, sa stabilité politique et son niveau de développement relativement satisfaisant, accueille des milliers de migrants relevant de cette catégorie et provenant presque exclusivement d'Afrique subsaharienne. Face à cette situation, les pouvoirs publics marocains ont adopté une politique migratoire prudente et politiquement opportuniste. Cela s'explique essentiellement par la nécessité de maintenir des équilibres dans un contexte régional et international extrêmement difficile. D'une part, et 
par souci d'améliorer son image sur la scène internationale, le Maroc a tenté d'envoyer des signaux positifs quant au traitement des flux migratoires, et ce, en abandonnant une gestion musclée au profit d'une gestion plus humanitaire et une assistance aux personnes fuyant les désastres économiques. D'autre part, il a réussi à utiliser une sorte de "diplomatie migratoire" afin de préserver et entretenir ses intérêts géopolitiques à la fois avec I'Union européenne et le reste du continent africain.

Trois enseignements peuvent être tirés de l'expérience marocaine en matière de traitement de la question des "réfugiés économiques". En premier lieu, il semble que le Maroc cherche à imiter les pays occidentaux en matière de gestion des flux migratoires et de la communication qui s'y rapporte, ce qui risque de créer un effet-contagion dans l'avenir. En effet, les témoignages des personnes régularisées et la perception globalement positive des médias risquent d'inciter de nouveaux migrants à tenter leur chance. En deuxième lieu, le fardeau social et sécuritaire des "réfugiés économiques" originaires d'Afrique subsaharienne commence à se faire sentir essentiellement dans les grandes villes, surtout avec la montée du chômage des jeunes Marocains et la difficile intégration des nouveaux arrivants dans une société marquée historiquement par le rejet des populations catégorisées comme «noires». En troisième lieu, un certain paradoxe apparait au niveau du découpage entre "réfugiés politiques" reconnus et "réfugiés économiques" non reconnus. En effet, même non encore régularisés, plusieurs médias ont relevé que la situation des premiers est nettement meilleure que celles des seconds (un niveau de vie relativement élevé). Pourtant, ce sont eux qui sont visés par la Convention de Genève, ce qui donne une certaine légitimité à notre position visant à faire reconnaitre le statut de «réfugiés économiques».

Nous estimons que la protection et l'atténuation de la souffrance des "réfugiés économiques" passent par la mise en œuvre d'une véritable feuille de route élaborée à l'échelle mondiale et tenant compte des spécificités nationales. En effet, les mouvements migratoires ont un caractère intercontinental. Les politiques devraient alors être conçues dans un cadre global et de solidarité internationale soutenue. C'est dans cette perspective que s'inscrit l'élaboration par le HCR d'un Pacte Global sur les réfugiés dont l'avant-projet a été publié le 31 janvier 2018. Celui-ci se veut une réponse intéressante, mais qui demeure assez vague, de la communauté internationale aux inquiétudes suscitées par l'ampleur et la sévérité des mouvements des réfugiés et les situations qui se prolongent. En dépit des bonnes intentions qui soutiennent ce projet, on déplore l'attachement de ses rédacteurs au texte de la Convention de Genève qui ignore la persécution économique comme motif réel de détermination du statut de réfugié. Certes, s'il est fait allusion aux migrations mixtes, qui incluent les "réfugiés économiques", ce texte en l'état parait insuffisant d'autant plus qu'aucun statut juridique ad hoc ne leur est précisément destiné. Par conséquent, aucune garantie n'est apportée quant à un traitement identique des dossiers et à l'absence d'opérations de tri aux frontières. On déplore également que ce Pacte ne soit pas un instrument juridiquement contraignant pour les États.

Le Pacte Global sur les réfugiés, de même que les autres stratégies et Conventions internationales dans le domaine de la migration n'auront d'effets réels que s'ils reconnaissent le statut de "réfugié économique" et s'insèrent en 
même temps dans des plans d'action à moyen et long terme, élaborés à l'échelle nationale et prenant en compte les spécificités et les contraintes locales. Une telle démarche est nécessaire pour mieux orienter les politiques migratoires vers les vraies priorités. Elle doit s'inscrire selon nous dans une logique d'audace et conduire à un débat constructif, impliquant toutes les parties prenantes et incluant un calendrier précis sur les procédures à suivre et les étapes à franchir pour atteindre les objectifs. Ceci est d'autant plus urgent et nécessaire que les flux des populations vont non seulement s'accentuer, mais surtout se recomposer en raison des contraintes imposées par la mondialisation et les changements environnementaux.

\section{Références bibliographiques}

Akoka Karen (2013) Le réfugié est une notion fabriquée au gré des priorités politiques, Interview effectuée par Carine Fouteau le 12 juin 2013, Medipart, [en ligne] consulté le 13/06/2017. URL : https://www.mediapart.fr/journal/france/120613/ le-refugie-est-une-notion-fabriquee-au-gre-des-priorites-politiques?onglet=full

Akoka Karen (2011) L'archétype rêvé du réfugié, Plein droit, 3 (90), pp. 13-16.

Alami M'Chichi Houria (2005) La migration dans la coopération UE-Maroc entre tentative de gestion institutionnelle eț pragmatisme, in Houria Alami M'Chichi, Bachir Hamdouch et Mehdi Lahlou Éds., Le Maroc et les migrations, Rabat, Fondation Friedrich Ebert, pp.13-40.

Alaoui Rachid (2013) Peut-on parler de diaspora marocaine?, Hommes \& migrations, 1303, pp. 7-15.

Alioua Mehdi (2005) La migration transnationale des Africains subsahariens au Maghreb : I'exemple de l'étape marocaine, Maghreb-Machrek, 185, pp. 37-57.

Barros Lucile, Lahlou Mehdi, Escoffier Claire, Pumares Pablo et Ruspini Paolo (2002) L'immigration irrégulière subsaharienne à travers et vers le Maroc, Cahiers de Migrations Internationales, 54F, Genève, Bureau International du Travail (BIT), $152 \mathrm{p}$.

Belaidi Nadia (2008) La lutte contre les atteintes globales à l'environnement : vers un ordre public écologique?, Bruxelles, Éditions Bruylant, 498 p.

Bernoussi Nadia et Benkeroum Mohamed (2017) Migrations internationales et justice constitutionnelle. Référendums et justice constitutionnelle, Annuaire international de justice constitutionnelle, pp. 421-440.

Betts Alexander (2013) Survival migration. Failed Governance and Crisis of Displacement, Ithaca/London, Cornell University Press, 256 p.

Beurrier Jean Pierre et Kiss Charles Kiss (2010) Droit international de l'environnement, Paris, Pédone, 598 p.

Blondel Marion (2014) Quelle protection pour les réfugiés "économiques"?, in Anne-Marie Tournepiche Éds., La protection internationale et européenne des réfugiés, Paris, Éditions A. Pedone, pp. 157-168.

Cambrézy Luc (2001) Réfugiés et exilés. Crise des sociétés, crise des territoires, Paris, Éditions des Archives contemporaines, 216 p. 
Cassarino Jean Pierre et Lavenex Sandra (2012) La gouvernance européenne des migrations dans la région méditerranéenne : la promesse d'un partenariat (équilibré)?, in L'Annuaire IEMed de la Méditerranée, Barcelona, Éditions de I'Institut européen de la Méditerranée (IEMed), pp.316-320.

CNDH (Conseil National des Droits de I'Homme) (2013) Étrangers et droits de I'Homme au Maroc : pour une politique d'asile et d'immigration radicalement nouvelle, Rapport thématique, Rabat, 10 p., [en ligne] consulté le 18/11/2017. URL : http://cndh.ma/sites/default/files/etrangers_et_droits_de_lhomme.pdf

FAO (Organisation des Nations unies pour I'alimentation et I'agriculture (2017) Vue d'ensemble régionale de la sécurité alimentaire et la nutrition. Le lien entre les conflits et la sécurité alimentaire et la nutrition : renforcer la résilience pour la sécurité alimentaire, la nutrition et la paix, Rapport, Accra, 116 p., [en ligne] consulté le 16/11/2017. URL : http://www.fao.org/3/a-i7967f.pdf

Gonin Patrick et Lassailly-Jacob Véronique (2002) Les réfugiés de I'environnement, une nouvelle catégorie de migrants forcés?, Revue Européenne des Migrations Internationales, 18 (2), pp. 139-160.

HCP (Haut Commissariat au Plan) (2017) Principaux résultats relatifs aux nouvelles thématiques couvertes par l'enquête nationale sur l'emploi, Rabat, 25 p., [en ligne] consulté le 22/06/2017. URL : https://www.hcp.ma/attachment/990273/

HCR (Agence des Nations Unies pour les réfugiés) (2016) "Réfugiés" et "migrants" - Questions fréquentes, [en ligne] consulté le 12/07/2017. URL : http:// www.unhcr.org/fr/news

HCR (Agence des Nations Unies pour les réfugiés) (2007) Convention et Protocole relatifs au statut des réfugiés, Genève, 54 p., [en ligne] consulté le 15/06/2017. URL : https://www.unhcr.org/fr/4b14f4a62.html\# ga=1.128288074.73 4145438.1442498391

Henry Sabine, Schoumaker Bruno and Beauchemin Cris (2004) The Impact of Rainfall on the First Out-Migration: A Multilevel Event-History Analysis in Burkina Faso, Population and Environnement, 25 (5), pp. 423-460.

Hinnant Lori (2018) Walk or die: Algeria strands 13.000 migrants in the Sahara, The Associated Press, [online] last checked on 20/09/2018. URL: https://apnews. com/9ca5592217aa4acd836b9ee091ebfc20

Khachani Mohamed (2010) Maroc : Migration, marché du travail et développement, Document de travail, Genève, Institut international d'études sociales, 68 p.

Magniny Véronique (1999) Les réfugiés de l'environnement : hypothèse juridique à propos d'une menace écologique, Thèse de Doctorat, Université Paris 1, 646 p.

McAdam Jane (2009) From Economic Refugees to Climate Refugees, Melbourne Journal of International Law, 10 (2), pp. 579-595.

Nations Unies (2016) Sûreté et dignité : gérer les déplacements massifs de réfugiés et de migrants, Rapport du Secrétaire général, [en ligne] consulté le 25/03/2017. URL : https://refugeesmigrants.un.org/sites/default/files/sg_report_ french.pdf

OECD (Organization for Economic Co-operation and Development) (2012) The Missing Piece: Improving International Support to the Peace Process, Paris, OCDE, $112 \mathrm{p}$. 
OUA (Organisation de I'Union africaine) (1974) Convention de I'OUA régissant les aspects propres aux problèmes de réfugiés en Afrique, [en ligne]. URL : http:// www.achpr.org/fr/instruments/refugee-convention/

Qassemy Halima (2014) Les enfants migrants à l'école marocaine. État des lieux sur l'accès à l'éducation des enfants migrants subsahariens au Maroc, Rapport mené pour le compte de I'ONG Oum ElBanine en collaboration avec la Fondation Terre des Hommes, 53 p. [en ligne] consulté le 10/08/2017. URL : https:// resourcecentre.savethechildren.net/sites/default/files/documents/rapport-les-enfants-migrants-et-lecole-marocaine_tamkine_migrants_v_finale.pdf

UNHCR (United Nations High Commissioner for Refugees) (2017) Global Trends: Forced Displacement in 2016, Geneva, 72 p, [online] last checked on 14/03/2017. URL: https://www.refworld.org/docid/594aa38e0.html

UNHCR (United Nations High Commissioner for Refugees) (2016) Refugee or migrant-Which is right?, [online] last checked on 11/03/2017. URL: http://www. unhcr.org/55df0e556.html

United Nations (2017) International Migration Report, New York, Department of Economic and Social Affairs, 38 p., [online] last checked on 12/04/2018. URL: http://www.un.org/en/development/desa/population/migration/publications/ migrationreport/docs/MigrationReport2017_Highlights.pdf

United Nations (2015) Trends in International Migration, 2015, Population facts, 4, 4 p., [online] last checked on 15/03/2017. URL: http://www.un.org/ en/development/desa/population/migration/publications/populationfacts/docs/ MigrationPopFacts20154.pdf

Wihtol de Wenden Catherine (2005) Atlas des migrations dans le monde, réfugiés ou migrants volontaires, Paris, Autrement, $79 \mathrm{p}$. 


\section{Brahim Elmorchid et Hind Hourmat Allah}

\section{Le Maroc face au défi des réfugiés économiques : quelle approche pour quelle gouvernance migratoire ?}

L'objectif de cet article consiste à mener une réflexion critique sur le mode de gouvernance des flux migratoires au Maroc. Une attention particulière est accordée à la place d'un nouveau type de migrants, celui que nous avons qualifié de "réfugiés économiques ". Deux principaux résultats se dégagent de cet exercice. En premier lieu, il semble urgent d'élargir le champ de définition de la Convention de Genève sur les réfugiés afin d'y inclure la persécution et la privation économique. Ceci est d'autant plus compréhensible que beaucoup de pays émetteurs de migrants connaissent aujourd'hui des crises économiques de plus en plus fréquentes et de plus en plus douloureuses. En deuxième lieu, l'arsenal juridique et institutionnel mis en place par le Maroc pour encadrer le droit des "réfugiés économiques " et des immigrés en général est assez riche. Toutefois, des dysfonctionnements graves et répétés persistent. Les politiques publiques migratoires dans leur ensemble se caractérisent par l'absence de visibilité et de cohérence susceptible d'inspirer des actions claires et concrètes. Elles demeurent sélectives, ponctuelles et politiquement opportunistes.

\section{The Challenge of Economic Refugees in Morocco: What Approach for which Migration Governance?}

This paper aims at conducting a critical reflection on the type of migration flows governance in Morocco. It will place a special focus on the position of a new type of migrants, namely economic refugees. Two main results emerge from this paper. First, there is an urgency to enlarge the definition of the Geneva Refugee Convention to include both economic persecution and deprivation. This is all the more understandable as many migrant sending countries are currently experiencing economic crises as much numerous as they are painful. Second, the legal and institutional arsenal set up by Morocco to regulate the rights of economic refugees and immigrants in general is quite rich. However, serious and repeated dysfunctions persist. Migration policies are characterized as a whole by the lack of visibility and coherence that should inspire clear and concrete actions. They remain selective, punctual, and politically opportunistic.

\section{Marruecos ante el reto de los refugiados económicos: ¿Qué aproximación para qué gobernanza migratoria?}

El propósito del presente trabajo es llevar a cabo una reflexión crítica sobre el modo de gobernanza de los flujos migratorios en Marruecos. Se presta especial atención a la importancia de un nuevo tipo de emigrantes, el de los refugiados económicos. Cabe destacar a este respecto dos principales resultados. En primer lugar, urge ampliar el campo de la definición del Convenio de Ginebra sobre los refugiados para incluir la persecución y la penuria económicas. Esto resulta tanto cuanto más comprensible que muchos de los países de procedencia de los emigrantes conocen hoy día crisis económicas cada vez más frecuentes y cada día más dolorosas. En segundo lugar, los mecanismos jurídicos e institucionales establecidos por Marruecos para enmarcar el derecho de los refugiados económicos y de los emigrados en general son bastante ricos. Sin embargo, persisten graves y frecuentes disfunciones. Las políticas migratorias se caracterizan en su conjunto por la ausencia de visibilidad y de coherencia. Siguen siendo selectivas, puntuales y políticamente oportunistas. 